\title{
Development of lentiviral gene therapy for retinitis pigmentosa
}

\author{
Makiko Uchiyama ${ }^{1}$, Tsutomu Ichiki ${ }^{1}$, Yoichi Naknishi ${ }^{1}$, Yasuhiro Ikeda ${ }^{2}$ \\ ${ }^{I}$ Center for clinical and translational research, Kyushu University Hospital, Japan, ${ }^{2}$ Department Ophthalmology, \\ Graduate School of Medical Sciences, Kyushu University, Japan
}

\section{Background}

Retinitis pigmentosa (RP) is an inherited and intractable disease causing blindness. Simian immunodeficiency virus (SIVagm) based vectors carrying human pigment epithelium derived factor (SIV-hPEDF) injecting into retinas was efficient and safe for long-lasting retinal gene transfer in non-clinical studies. We are developing a new lentiviral gene therapy for RP in collaboration with researchers, biotech and academic research organization.

Several gene therapies have been approved in EMA, and FDA recently approved Luxturna, an AAV based gene therapy for retinal dystrophy. However, no gene therapy product has been approved in Japan yet. Therefore, to discuss the regulatory requirements and process is important.

\section{Methods and results}

We have completed a phase 1 study for 5 RP patients, and next phase 1/2a study will be initiated in summer 2018. Prior to these studies, protocols, quality and safety profile of vectors were reviewed by the regulatory office in Japan. Regulatory discussions were divided into 4 parts; quality of materials, quality and comparability of product, safety and study design. Providing the biosafety data of vectors was particularly required based on the current regulations and Cartagena protocol.

\section{Conclusions}

Regard to safety issues, competency of vectors and assessment method of efficacy, we have spent most of time for regulatory discussion. We will soon initiate a phase $1 / 2$ a study to assess the safety and efficacy of SIV-hPEDF for RP. Gene therapy is still challenging in Japan but will provide therapeutic options. 